\title{
Leukoaraiosis and cause of death: a five year follow up
}

\author{
S Tarvonen-Schröder, T Kurki, I Räihä, L Sourander
}

\begin{abstract}
The causes of death of 127 patients, who had undergone CT examination of the brain in 1989, were investigated. The CT was re-evaluated. Twenty five patients were excluded because of pathological findings on CT other than leukoaraiosis (LA), infarction, or their combination or, because of a specific known aetiology for LA. Of the remaining 102 patients, 25 had pure $L A, 18$ had pure infarction, 37 had LA combined with infarction (cLA), and 22 had a normal CT. The mean time between the CT and death was 1.8 (SD 1.5) years. A vascular cause of death was clearly associated with LA and with the severity of LA. Patients with pure LA had a vascular cause of death as often as those with pure infarction and those with LA combined with infarction. These groups differed significantly from each other when comparing the occurrence of cerebrovascular, cardiovascular and other vascular causes of death. The results suggest that LA on CT is more likely to be associated with a cardiovascular cause of death, and pure infarction is more often associated with a cerebrovascular death.
\end{abstract}

$(\mathfrak{F}$ Neurol Neurosurg Psychiatry 1995;58:586-589)

Keywords: cause of death; computed tomography; leukoaraiosis; vascular death

Leukoaraiosis (LA) is a non-specific radiological sign in the cerebral white matter shown by CT or MRI. ${ }^{1}$ The clinical relevance and aetiopathogenesis of these white matter lesions are far from clear. They may be heterogeneous in origin, an end stage due to various mechanisms, ${ }^{2}$ or a manifestation of a vascular process, ${ }^{2-4}$ but most likely not an end point of it. ${ }^{3-5}$ LA can be found in normal elderly subjects ${ }^{2}$ and in those with no evidence of vascular disease ${ }^{6}$ as well as in pathological conditions. In some studies LA has been associated with various cerebrovascular risk factors $^{2}$ including both hypertension ${ }^{25}$ and hypotension $^{7-9}$; LA itself has been found to be a risk factor for future strokes, ${ }^{10-12}$ for development of clinically relevant cerebrovascular disease, ${ }^{1113}$ and for poor overall prognosis. ${ }^{12} 14$ Causes that have been suggested for LA include Wallerian degeneration secondary to neuronal death in association with Alzheimer's disease ${ }^{15}$ and also in association with cortical infarction, ${ }^{16}$ but other researchers disagree. ${ }^{317}$ Hypoperfusion associated with LA in a number of perfusion studies $^{13}{ }^{18}$ may be not only causal, but may also be a consequence of it. ${ }^{15}$ As well as the common neuropathological finding of hyaline vascular stenosis in association with $\mathrm{LA},{ }^{378}$ amyloid angiopathy has been considered a possible pathogenetic basis for it $^{1418} 19$ - but not unanimously. ${ }^{378}$

To test further the significance of leukoaraiosis on CT, we investigated its association with the cause of death, especially from vascular disease.

\section{Patients and methods PATIENTS}

In the geriatric department of Turku City Hospital, CT examination of the brain for various reasons was performed in 253 patients from January to December 1989 . It was standard practice in the department that a neurologist examined patients with neurological or mental problems and decided whether CT examination of the brain was necessary. It was possible to obtain sufficient data on 208 patients, of whom 25 were excluded because of a specific known aetiology for LA or pathology on CT other than LA, or infarction, or their combination. At the time of this investigation (May 1994) 102 patients had died. Of these 62 had LA; 25 as the sole pathological finding on CT-that is, pure LA and 37 with LA combined with infarctionthat is, combined LA. Of the 40 patients who did not have LA lesions, 18 had infarction as the sole pathological finding on CT, (pure infarction) and 22 had a normal CT finding. The mean time between the CT and death was $1.8(\mathrm{SD} 1.5)$ years.

\section{CLINICAL INFORMATION}

The hospital records including the detailed medical history of the patients, death certificates, and necropsy reports were interpreted by the same neurologist (ST-S) without knowing whether LA was present, but knowing other findings on CT. Necropsies were done on $47(46 \%)$ of the 102 patients. The diagnosis of dementia was based on DMS-III$R^{20}$ The patients were divided into four groups: (a) no dementia; (b) Alzheimer's disease diagnosed in accordance with the NINCDS-ADRDA criteria for probable Alzheimer's disease ${ }^{21}$; $(c)$ vascular dementia diagnosed in accordance with the NINDSAIREN criteria 22 for "probable VAD" and the ischaemic score of Hachinski ${ }^{23}$; (d) other 
Table 1 Demographic data on patients with pure $L A$ ( $p L A)$, pure infarction ( $p$ Inf), combined $L A$ and infarction ( $C L A)$, and a normal $C T$ finding ( $N C T)$

\begin{tabular}{lcccll}
\hline & $\begin{array}{c}p L A \\
(n=25)\end{array}$ & $\begin{array}{c}p \operatorname{Inf} \\
(n=18)\end{array}$ & $\begin{array}{l}c L A \\
(n=37)\end{array}$ & $\begin{array}{l}n C T \\
(n=22)\end{array}$ & Pvalue \\
\hline Mean age (y (SD)) & $85 \cdot 9(1 \cdot 3)$ & $75 \cdot 2(2 \cdot 1)$ & $82 \cdot 5(1 \cdot 0)$ & $73 \cdot 1(1 \cdot 6)$ & $0 \cdot 0000$ \\
Sex (women) & $20(80)$ & $8(44)$ & $26(70)$ & $17(77)$ & NS \\
Cognitive status: & & & & & \\
No dementia & $6(24)$ & $8(44)$ & $6(16)$ & $8(36)$ & NS \\
AD & $2(8)$ & $0(0)$ & $2(5)$ & $4(18)$ & NS \\
VAD & $14(56)$ & $10(56)$ & $29(78)$ & $5(23)$ & $0 \cdot 0006$ \\
Other dementia & $3(12)$ & $0(0)$ & $0(0)$ & $5(23)$ & $0 \cdot 0073$ \\
\hline
\end{tabular}

Except for age, values are numbers of patients (\%)

$\mathrm{AD}=$ probable Alzheimer's disease; $\mathrm{VAD}=$ probable vascular dementia or combined vascular and degenerative dementia. dementia (aetiology other than Alzheimer's disease or vasicular dementia-for instance, alcoholism, previous trauma, or schizophrenia). The diagnosis of the primary cause of death was based on the International Statistical Classification of Diseases, Injuries, and Causes of Death (ICD-9). ${ }^{24}$

\section{COMPUTED TOMOGRAPHY}

Findings from CT were interpreted by the same neuroradiologist (TK), who was not aware of the clinical data. A Toshiba $80 \mathrm{~A}$ scanner had been used in all examinations. The CT had been obtained from the base to the vertex of the brain. The slice thickness was $10 \mathrm{~mm}$ with the exception of the base of the brain where it was $5 \mathrm{~mm}$. The evaluation was done from hard copy $x$ ray films.

Bilateral symmetric confluent areas with reduced CT attenuation contiguous with the margins of the lateral ventricles were designated as LA lesions. Areas with decreased attenuation of the white matter could be located at the margins of the frontal and occipital horns of the lateral ventricles or they may have extended towards the centrum semiovale. Lesions could be irregular or patchy but mostly they tended to be uniform and diffuse.

The distribution of LA was divided into three areas: frontal, central, and occipital. The extent of LA in these areas was graded on visual impression as follows: 0 , no $L A ; 1$, under $1 / 4$ of the area; $2,1 / 4-1 / 2$ of the area, and 3 , over $1 / 2$ of the area. The severity of LA was graded from 1 to 9 on the basis of the extent of the lesions in the three areas. Lesions graded from 1 to 5 were considered mild to moderate, and lesions graded over 5 were considered severe.

Focal changes suggesting previous cerebral infarction were also registered. Central lacunae and porencephalic cysts, and localised

Table 2 Occurrence of cerebrovascular, cardiovascular, and other vascular causes of death in patients with pure $L A$ ( $p L A)$, pure infarction ( $p$ Inf), combined $L A$ and infarction (cLA), and a normal CT finding ( $n C T)$

\begin{tabular}{lllll}
\hline \multicolumn{5}{c}{ Primary cause of death (\%) } \\
\cline { 2 - 5 } Finding on CT & Cerebrovascular & Cardiovascular & Other vascular & Total vascular \\
\hline pLA $(\mathrm{n}=25)$ & $2(8)$ & $12(48)$ & $3(12)$ & $17(68)^{\star}$ \\
mild/moderate $(\mathrm{n}=18)$ & $0(0)$ & $8(44)$ & $3(17)$ & $11(61)$ \\
severe $(\mathrm{n}=7)$ & $2(29)$ & $4(57)$ & $0(0)$ & $6(86) \dagger$ \\
$\mathrm{pInf}(\mathrm{n}=18)$ & $10(56)$ & $2(11)$ & $3(17)$ & $15(83) \neq$ \\
cLA $(\mathrm{n}=37)$ & $16(43)$ & $8(22)$ & $5(13)$ & $29(78) \S$ \\
nCT $(\mathrm{n}=22)$ & $3(13)$ & $1(15)$ & $3(14)$ & $7(32)$
\end{tabular}

$\star \mathrm{P}=0.01 v$ patients with $\mathrm{nCT} ; \mathrm{\dagger P}=0.02 v$ patients with $\mathrm{nCT} ; \ddagger \mathrm{P}=0.001 v$ patients with $\mathrm{nCT} ; \oint \mathrm{P}=0.0004 v$ patients with $\mathrm{nCT}$. atrophies with a distribution corresponding to major brain arteries were considered postinfarction lesions. To simplify matters, the different types of infarctions (for example, lacunes and large cortical infarcts) were not differentiated even though they may have been consequences of different pathophysiological processes.

\section{STATISTICAL ANALYSIS}

The Mann-Whitney test was used for comparison of means of severity (extent) of LA between the groups with pure LA and combined LA. The Kruskall-Wallis test was used for multiple comparisons of means of the Hachinski score among the four study groups. A one way analysis of variance (ANOVA) test was used for multiple comparisons of mean ages at death among the four groups, and a $t$ test with Bonferroni correction for paired comparisons. Associations between categorial variables were analysed with Pearson's $\chi^{2}$ test. When the counts in the cells were less than 5, Fisher's two tailed exact test was used. Associations between categorical (causes of death) and continuous (age at death) variables were analysed by dichotomous and multinomial logistic regression analysis. The limit of significance used was $\mathrm{P}<0.05$.

\section{Results}

Table 1 shows demographic data on the patients in the four different study groups; LA (both pure LA and combined LA as compared with normal CT) was strongly associated with age, but pure infarction was not. The cause of death, however, vascular or nonvascular on the one hand and the type of vascular death (cerebrovascular, cardiovascular, and other vascular) on the other were not associated with age or with sex. Thus adjustment for age or sex among the different groups was not needed.

Table 2 shows that a vascular cause of death was clearly associated with pure LA and the severity of pure LA. Patients with severe pure LA had a vascular cause of death as often as those with pure infarction. The severity (extent) of LA did not differ between the groups with pure LA (mean $4 \cdot 3$ (SD 2.5)) and combined LA (3.9 (SD 2.3)). The four groups differed significantly from each other, however, when comparing the type of vascular death (table 2). The most frequent cause of death in the pure LA group was a cardiovascular disorder $(48 \%)$, but a cerebrovascular disorder was most frequent in the pure infarction group (56\%); the group with combined LA was between these two. Conspicuously, the occurrence of a cerebrovascular cause of death was not higher in the pure LA group than in the normal CT group. The results did not change when only those necropsied were analysed.

Of the 12 patients with a cardiovascular cause of death in the pure LA group, nine (75\%) had a symptomatic cardiac disease before the terminal stage (coronary artery disease or congestive heart failure). Of those 
Table 3 Occurrence of coronary artery disease (CAD), cardiac failure, and Hachinski ischaemic score $>7$ in patients with pure $L A$ ( $p L A)$, pure infarction ( $P$ Inf), combined $L A$ and infarction ( $C L A)$, and a normal $C T$ finding $(n C T)$

\begin{tabular}{llllll}
\hline & $\begin{array}{l}p L(n=25) \\
n(\%)\end{array}$ & $\begin{array}{l}p \operatorname{Inf}(n=18) \\
n(\%)\end{array}$ & $\begin{array}{l}c L A(n=37) \\
n(\%)\end{array}$ & $\begin{array}{l}n C T(n=22) \\
n(\%)\end{array}$ & P value \\
\hline CAD & $10(40)$ & $8(44)$ & $15(40)$ & $10(45)$ & NS \\
Cardiac failure & $15(60)$ & $6(33)$ & $16(43)$ & $3(14)$ & $<0.01$ \\
Hachinski score $>7$ & $15(60)$ & $18(100)$ & $30(81)$ & $13(59)$ & $<0.01$ \\
\hline
\end{tabular}

without a previous diagnosis of a cardiac disease, two had vascular dementia and one had Alzheimer's disease.

There were no significant differences among the groups in the occurrence of premortem symptomatic coronary artery disease, but cardiac failure did differentiate between them (table 3). The ischaemic score of Hachinski $(>7)$ also differentiated these groups from each other (mean values $7 \cdot 3$ (SD 3.5) for pure LA, 11.6 (SD 2.5) for pure infarction, 10.9 (SD 3.9) for combined LA, and $7 \cdot 4$ (SD $4 \cdot 1)$ for normal CT $(P=$ $0 \cdot 0000)$ ).

\section{Discussion}

Hachinski and coworkers ${ }^{1}$ created the descriptive radiological label "leukoaraiosis", which may be replaced in the future with greater understanding, as subclassifications of these deep white matter changes based on aetiopathogenetic factors will inevitably arise. Today, the nature and relevance of these changes in white matter are still far from being solved and a subject of much controversy. Various investigators have studied the clinical and pathological correlates of LA from different points of view, ${ }^{24}$ but there is a conspicuous lack of studies focused on the overall causes of death of those with LA.

In the present study, a vascular cause of death was clearly associated with pure LA of unknown aetiology. A vascular cause of death was as frequent in patients with severe pure LA as it was in patients with brain infarction or the combination of these two conditions. The frequency of deaths due to stroke, however, was not higher in patients with pure LA than in patients with a normal CT finding-as it was in the other two groups. Instead, a cardiovascular disorder was the leading cause of death in patients with pure LA. In this respect, patients with combined LA were in between the groups with pure LA and those with pure infarction.

The reliability of these results may be questioned because of the retrospective nature of this study and the fact that only half of the patients were necropsied. The reliability of hospital records and death certificates in Finland is, however, very high. Furthermore, when excluding the patients not necropsied, the results did not change. The extrapolation of these results to other populations has to be done with caution, however, because the subjects were selected, mostly elderly patients in hospital. The fact that this study was based on CT and not MRI may raise the question of the sensitivity of the method; CT cannot detect abnormalities as small as those found on MRI. Small MRI lesions, however, are of more heterogeneous origin and do not necessarily permit differentiation among the different underlying pathological entities. ${ }^{2}$ Although not denying the usefulness of MRI, it has been suggested that CT should be considered a more useful method to evaluate clinical radiological correlates in dementia. ${ }^{11}$

In patients with transient ischaemic attacks or minor stroke LA has been associated with an extra risk of future stroke, independent of other risk factors. ${ }^{1012}$ A correlation between the presence of LA and cerebrovascular disease has been suggested, implying that LA may provide evidence of "silent" cerebrovascular disease. ${ }^{13}$ The present study suggests that a risk of fatal cardiac disease exists in patients with LA. In fact, Miyao and coworkers $^{12}$ found cardiac disease to be the major cause of death $(40 \%)$ in $10 \mathrm{LA}$ positive patients with lacunar infarction. Obviously, fatality for LA due to direct neurological sequelae is negligible, as it is in the case of lacunar infarctions. The complications of extracranial arteriosclerosis and cardiac diseases, however, threaten patients with LA as they do those with true brain infarctions. If, as has been suggested, focal brain infarctions also develop in association with severe LA, ${ }^{2}$ the probability of fatal cerebral stroke seems to increase. The results of the present study support the idea that arteriosclerotic vascular disease is the common pathogenetic pathway for both LA and cerebral infarction ${ }^{525}$ with infarction being the end point of the process, not LA. ${ }^{4}$

In this study, however, the patients with combined LA did not have a more severe degree of LA and were not older than those with pure LA. Instead, the patients with pure and combined LA were significantly older than those with pure infarction. This suggests that LA is not just a simple continuum of a process leading to true infarction. In addition, the Hachinski score was equal in the pure LA and normal CT groups, and significantly higher in the other two groups with infarction, which also fails to support a simple multiinfarct aetiology of pure LA. Although both conditions have been shown to correlate with arteriosclerosis and share many of the same risk factors, including hypertension, there seem to be some differences between them. The most common pathogenetic pathway for infarctions is thromboembolism, ${ }^{2}$ whereas the pathway for LA in these elderly patients seems to be haemodynamic failure due to cardiovascular disorders. In a previous study we have shown that hypotension and cardiac failure are associated with LA later in life. ${ }^{9}$ These findings are consistent with those of Brun and Englund. ${ }^{717}$ The fact that vascular mechanisms frequently overlap, ${ }^{26}$ often makes clinical differential diagnosis difficult.

In conclusion, in the present study LA was associated with a cardiovascular rather than a cerebrovascular cause of death. This finding suggests that LA is a manifestation of a more general vascular disorder than simple 
cerebrovascular disease. The association between heart failure and LA suggests that haemodynamic failure with subsequent cerebral hypoperfusion contributes to the pathogenesis of LA.

We are grateful to Hans Helenius, for his expert advice in biostatistics and Ms Pirjo Piekka for her skilled secretarial assistance.

1 Hachinski VC, Potter P, Merskey H. Leuko-araiosis. Arch Neurol 1987;44:21-3.

2 Erkinjuntti T, Hachinski VC. Rethinking vascular dementia. Cerebrovascular Diseases 1993;3:3-23.

3 Englund E, Brun A, Alling C. White matter changes in dementia of Alzheimer's type. Biochemical and neuropathological correlates. Brain 1988;111:1425-39.

4 Wallin A, Blennow K. Pathogenetic basis of vascular dementia. Alzheimer's Disease and Associated Disorders 1991;5:91-102.

5 Wallin A, Blennow $\mathrm{K}$, Uhlemann $\mathrm{C}$, Långström $\mathrm{G}$, Gottfries CG. White matter low attenuation on computed tomography in Alzheimer's disease and vascular puted tomography in Alzheimer's disease and vascular dementia-diagnostic and parol Scand 1989;80:518-23.

6 George $\mathrm{AE}$, de Leon MJ, Genters CI, et al. Leukoencephalopathy in normal and pathologic aging: 1. CT of brain lucencies. AfNR Am $\mathcal{F}$ Neuroradiol 1986;7:561-6.

7 Brun A, Englund E. A white matter disorder in dementia of Alzheimer type: A pathoanatomical study. Ann Neurol 1986;19:253-62.

8 Englund E, Brun A, Gustafson L. A white matter disease in dementia of Alzheimer's type-clinical and neuropathological correlates. International fournal of Geriatric Psychiatry 1989;4:87-102.

9 Räihä I, Tarvonen S, Kurki T, Rajala T, Sourander L. Relationship between vascular factors and white matter low attenuation of the brain. Acta Neurol Scand 199 attenuation

10 van Swieten JC, Kappelle LJ, Algra A, van Latum JC, Koudstaal PJ, van Gijn J, for the Dutch TIA Trial Study Group. Hypodensity of the cerebral white matter in patients with transient ischemic attack or minor stroke: influence on the rate of subsequent stroke. Ann Neurol 1992;32:177-83.

11 Lopez OL, Becker JT, Rezek D, et al. Neuropsychiatric correlates of cerebral white-matter radiolucencies in probable Alzheimer's disease. Arch Neurol 1992;49:828-34.

12 Miyao S, Takano A, Teramoto J, Takahashi A.
Leukoaraiosis in relation to prognosis for patients with lacunar infarctions. Stroke 1992;23:1434-8.

13 Fazekas F, Niederkorn K, Schmidt R, et al. White matter signal abnormalities in normal individuals: correlation with carotid ultrasonography, cerebral blood flow measurements, and cerebrovascular risk factors. Stroke 1988;19:1285-8.

14 Janota I, Mirsen TR, Hachinski VC, Lee DH, Merskey H. Neuropathologic correlates of leuko-araiosis. Arch Neurol 1989;46:1124-8.

15 Leys D, Pruvo JP, Parent M, et al. Could Wallerian degeneration contribute to "leuko-araiosis" in subjects free of any vascular disorder? $\mathcal{f}$ Neurol Neurosurg Psychiatry 1991;54:46-50.

16 Leifer D, Buonnanno FS, Richardson EP. Clinicopathologic correlations of cranial magnetic resonance imaging of periventricular white matter. Neurology 1990;40:911-8.

17 Englund E, Brun A. White matter changes in dementia of Alzheimer's type: the difference in vulnerability between cell compartments. Histopathology 1990;16:433-9.

18 Kawamura J, Meyer JS, Ichijo M, Kobari M, Terayama Y, Weathers S. Correlations of leuko-araiosis with cerebral atrophy and perfusion in elderly normal subjects and demented patients. $f$ Neurol Neurosurg Psychiatry demented patient

19 Tabaton M, Caponnetto C, Mancardi G, Loeb C. Amyloid beta protein deposition in brains from elderly subjects with leukoaraiosis. F Neurol Sci 1991;106:123-7.

20 American Psychiatric Association. Diagnostic and statistical manual of mental disorders. Washington DC: American Psychiatric Association, 1987.

21 McKhann G, Drachman D, Folstein M, Katzman R, Price D, Stadlan EM. Clinical diagnosis of Alzheimer's disease: report of the NINCDS-ADRDA Work Group under the auspices of Department of Health and Human Services Task Force on Alzheimer's disease. Neurology 1984;34:939-44

22 Roman GC, Tatemichi TK, Erkinjuntti T, et al. Vascular dementia: diagnostic criteria for research studies. Report of the NINDS-AIREN International Workshop. Neurology 1993;43:250-60.

23 Hachinski VC, Iliff LD, Phil $M$, et al. Cerebral blood flow in dementia. Arch Neurol 1975;32:632-7.

24 International Statistical Classification of Diseases, Injuries, and Causes of Death (ICD-9, Finnish version) Geneva: World Health Organisation, 1987

25 Erkinjuntti T, Ketonen L, Sulkava R, Vuorialho M, Palo J. $\mathrm{CT}$ in the differential diagnosis between Alzheimer's disease and vascular dementia. Acta Neurol Scand 1987;75:262-70.

26 Erkinjuntti T. Types of multi-infarct dementia. Acto Neurol Scand 1987;75:391-9. 